\title{
On Tutte polynomial expansion formulas in perspectives of matroids and oriented matroids
}

\author{
Emeric Gioan*,a \\ ${ }^{a}$ CNRS, LIRMM, Université de Montpellier, France
}

\begin{abstract}
We introduce the active partition of the ground set of an oriented matroid perspective (or quotient, or strong map) on a linearly ordered ground set. The reorientations obtained by arbitrarily reorienting parts of the active partition share the same active partition. This yields an equivalence relation for the set of reorientations of an oriented matroid perspective, whose classes are enumerated by coefficients of the Tutte polynomial, and a remarkable partition of the set of reorientations into boolean lattices, from which we get a short direct proof of a 4 -variable expansion formula for the Tutte polynomial in terms of orientation activities. This formula was given in the last unpublished preprint by Michel Las Vergnas; the above equivalence relation and notion of active partition generalize a former construction in oriented matroids by Michel Las Vergnas and the author; and the possibility of such a proof technique in perspectives was announced in the aforementioned preprint. We also briefly highlight how the 5-variable expansion of the Tutte polynomial in terms of subset activities in matroid perspectives comes in a similar way from the known partition of the power set of the ground set into boolean lattices related to subset activities (and we complete the proof with a property which was missing in the literature). In particular, the paper applies to matroids and oriented matroids on a linearly ordered ground set.
\end{abstract}

Dedicated to Michel Las Vergnas' spirit.

\section{Introduction}

The paper deals with (oriented) matroid perspectives on a linearly ordered ground set, with the Tutte polynomial in terms of subset activities, and with the Tutte polynomial in terms of orientation activities. Let us situate these aspects with respect to the literature, and present the contributions of the paper. Formal definitions and general statements are given in the next sections.

An ordered pair $(M, N)$ of (oriented) matroids forms a perspective (also called morphism, or quotient, or strong map in the literature, up to unimportant variants), when they satisfy a certain structural relationship, which is notably consistent with linear maps in (real) vector spaces, and with (directed) graph homomorphisms in graphs. See [Kun86] or [Oxl11, Section 7.3] (see also [LV80, LV84]) in matroids, and BLVS $^{+}$99, Section 7.7] in oriented matroids. The Tutte polynomial

*Email: emeric.gioan@lirmm.fr 
of such a perspective has been defined in [LV80, LV99] in terms of rank functions as:

$$
t(M, N ; x, y, z)=\sum_{A \subseteq E}(x-1)^{r(N)-r_{N}(A)}(y-1)^{|A|-r_{M}(A)} z^{r(M)-r(N)-\left(r_{M}(A)-r_{N}(A)\right)} .
$$

Ever since, this polynomial has been mainly studied by Michel Las Vergnas in a series of papers, including [LV84, LV12, LV13]. See [Gioa] for a recent survey. In the particular case where $M=N$, one retrieves the usual Tutte polynomial of the matroid $M$.

The main novelty of the present paper concerns oriented matroid perspectives. Let us give a glimpse in terms of a single oriented matroid $M$ on a linearly ordered set $E$. The Tutte polynomial of $M$ is known to have the following expression [LV84]:

$$
t(M ; x, y)=\sum_{A \subseteq E}\left(\frac{x}{2}\right)^{o^{*}\left(-{ }_{A} M\right)}\left(\frac{y}{2}\right)^{o\left(-{ }_{A} M\right)}
$$

where $o^{*}\left(-{ }_{A} M\right)$, resp. $o\left(-{ }_{A} M\right)$, is the dual orientation activity, resp. orientation activity, of the oriented matroid $-{ }_{A} M$, which counts smallest elements of positive cocircuits, resp. circuits. See LV84, GLVa] for interpretations and applications of these orientation activity parameters. In LV12], a 4-variable expansion is given:

$$
t(M ; x+u, y+v)=\sum_{A \subseteq E} x^{\theta_{M}^{*}(A)} u^{\bar{\theta}_{M}^{*}(A)} y^{\theta_{M}(A)} v^{\bar{\theta}_{M}(A)}
$$

where the parameters used for exponents refine orientation activities, depending on $M$ and $A$, not only on $-{ }_{A} M$. Actually, both [LV84] and LV12] work at the level of oriented matroid perspectives (see general statements in Section 21), and a detailed example is given in [LV12]. The proof proposed in [LV12] is by deletion/contraction and is rather technica 1]. Also, it is suggested that an alternative proof could be obtained more structurally, using active partitions and activity classes of oriented matroids addressed in [Gio02, GLV05, GLVb, GLV07, GLVa] (see also [Giob] for an overview).

In Section 2, we give such a short direct proof. Furthermore, we also give it at the level of oriented matroid perspectives. For that purpose, we first introduce the active partition of the ground set of an oriented matroid perspective on a linearly ordered ground set. Then, we show that the reorientations obtained by arbitrarily reorienting parts of the active partition share the same active partition, a result which generalizes the same property known for oriented matroids. This yields an equivalence relation for the set of reorientations, whose classes are enumerated by coefficients of the Tutte polynomial, and a remarkable structural partition of this set into boolean lattices called activity classes. The enumerative counterpart of this partition turns out to be exactly the aforementioned 4-variable expansion formula for the Tutte polynomial. Various corollaries are derived, such as enumerating remarkable subsets of reorientations by Tutte polynomial evaluations.

\footnotetext{
${ }^{1}$ Let us warn the reader that [LV12] is an unpublished preliminary preprint, containing some inaccuracies. For instance, LV12, Equation (4) page 3] is not correct (the parameters $c r$ and $n l$ are missing, see [GT90, LV13]). More importantly, the definition of the refined active bijection given at the end of [LV12, page 20] is not correct: it is not complete and given with a wrong parameter correspondence. The correct definition and correspondence are given in GLVb, GLVa], consistently with the definitions previously given in Gio02, GLV07].
} 
As far as matroids are concerned, for a matroid $M$ on a linearly ordered set $E$, the Tutte polynomial has the two well-known following expressions [Tut54, Cra69]:

$$
t(M ; x, y)=\sum_{A \subseteq E}(x-1)^{r(M)-r(A)}(y-1)^{|A|-r(A)}=\sum_{B \text { basis }} x^{\iota(B)} y^{\varepsilon(B)}
$$

where $\iota(B)$, resp. $\varepsilon(B)$, is the internal, resp. external, activity of the basis $B$. A striking result is that those two expressions can be unified under the following 4-variable expansion:

$$
t(M ; x+u, y+v)=\sum_{A \subseteq E} x^{\iota(A)} u^{r(M)-r(A)} y^{\varepsilon(A)} v^{|A|-r(A)},
$$

where the notions of activities are extended to subsets. This result has been shown for matroids in [GT90] (actually in a more general form). It has been generalized in [LV13] to a 5-variable expansion formula the Tutte polynomial of a matroid perspective, recalled below as Theorem 12 , Let us mention that, using suitable variable specifications, one can obtain further expressions for the Tutte polynomial, see a list in GT90] completed in [LV13].

As precisely addressed in [LV13], the notion of subset activities is related to the following classical result: the set $2^{E}$ of subsets of $E$ is partitioned into boolean intervals of type [ $B \backslash$ $\operatorname{Int}(B), B \cup \operatorname{Ext}(B)]$, where $B$ ranges over all bases of $M$ (and where $\operatorname{Int}(B), \operatorname{resp} . \operatorname{Ext}(B)$, denotes the set of internally, resp. externally, active elements w.r.t. $B)$. This result, known for matroids from [Cra69], generalizes to matroid perspectives. Actually, it can be obtained using an even more general combinatorial construction [Daw81], which applies to matroids and matroid perspectives as well, as detailed in [LV13] (see also [Bjö92, LV01] for related results in matroids, [GM97] for an independent generalization to greedoids, and [BGdON09] for an inverse property in matroids).

In Section 3, placed at the level of matroid perspectives, we sum up this setting and highlight how the above expansion formula can be directly derived as the enumerative counterpart of the aforementioned fundamental structural partition of the power set into intervals. The proof in [LV13] uses this partition in an indirect way, passing through Tutte polynomial derivatives. Furthermore, a property of this partition is missing in [LV13], though required for the proof and implicitly used. Here, in Proposition [11, we explicitly state and prove this property, hence completing [LV13].

Finally, the point of the paper is to complete [LV12, LV13] and to show how each Tutte polynomial expansion formula is the enumerative counterpart of a remarkable structural partition of the power set into boolean lattices, arising as soon as the ground set is linearly ordered. The initial formula enumerates the boolean lattices each as an individual part, and the expansion formula with two more variables enumerates in addition all subsets within these boolean lattices.

From the stuctural viewpoint, let us mention that active partitions in oriented matroids have further remarkable properties, and that the active bijection transforms the formulas in terms of orientation and subset activities into each other, while transforming the boolean lattices of the two constructions into each other, e.g. see [Gio02, GLV05, GLVb, GLV07, GLVa]. The generalization of such properties and bijections to oriented matroid perspectives is work in progress.

\section{Building on orientation activities in oriented matroid perspectives}

The reader is referred to $\left.\mathrm{BLVS}^{+} 99\right]$ for background on oriented matroids, and in particular to $\left[\right.$ BLVS $^{+}$99, Section 7.7] for details on oriented matroid perspectives. Unless it is ambiguous, we 
usually use the same notation for a signed set and its support. Let us recall that two signed sets are conformal if they have the same signs on their intersection, and that a vector, resp. covector, in an oriented matroid can be defined as a conformal union of circuits, resp. cocircuits. An ordered pair $(M, N)$ of oriented matroids on the same ground set form an oriented matroid perspective (also called strong map or quotient map), denoted $M \rightarrow N$, if one of the following equivalent properties holds:

- any circuit of $M$ is a vector of $N$;

- any vector of $M$ is a vector of $N$;

- any cocircuit of $N$ is a covector of $M$;

- any covector of $N$ is a covector of $M$;

- no circuit of $M$ and cocircuit of $N$ have a non-empty conformal intersection 3 .

In particular, for an oriented matroid $M, M \rightarrow M$ is an oriented matroid perspective. Actually, if $M \rightarrow N$ is an oriented matroid perspective and $r(M)=r(N)$ then $M=N$. Moreover, if $M \rightarrow N$ is an oriented matroid perspective then $N^{*} \rightarrow M^{*}$ is also an oriented matroid perspective. Let us mention that the underlying matroids form a matroid perspective, and that, in contrast, an oriented matroid perspective is not equivalent to an extension followed by a contraction (see Section $\underline{3}$ ).

Let $M$ be an oriented matroid on a linearly ordered set $E$. Following [LV84], we denote

$$
\begin{aligned}
O(M) & =\{\min (C) \mid C \text { positive circuit of } M\} \\
O^{*}(M) & =\{\min (C) \mid C \text { positive cocircuit of } M\} .
\end{aligned}
$$

The sets $O(M)$ and $O^{*}(M)$ are respectively known as the sets of active and dual-active elements of $M$. The parameters $|O(M)|$ and $\left|O^{*}(M)\right|$ are respectively known as the (orientation) activity and dual (orientation) activity of $M$. One can observe that $O^{*}(M)=O\left(M^{*}\right)$, that $O(M) \cap O^{*}(M)=\emptyset$, that $O(M)=\emptyset$ if and only if $M$ is acyclic, and that $O^{*}(M)=\emptyset$ if and only if $M$ is totally cyclic.

Let $M \rightarrow N$ be an oriented matroid perspective on a linearly ordered set $E$. By [LV84], its Tutte polynomial satisfies

$$
t(M, N ; x, y, 1)=\sum_{A \subseteq E}\left(\frac{x}{2}\right)^{\left|O^{*}\left(-{ }_{A} N\right)\right|}\left(\frac{y}{2}\right)^{\left|O\left(-{ }_{A} M\right)\right|} .
$$

Note that, in particular, $t(M, N ; 0,0,1)$ is equal to the number of subsets $A$ of $E$ such that $-{ }_{A} M$ is acyclic and $-{ }_{A} N$ is totally cyclic (see [LV84] for some interpretations of this special evaluation).

\footnotetext{
${ }^{2}$ We choose to denote $M \rightarrow N$ rather than $M \rightarrow M^{\prime}$ which is commonly used in references on (oriented) matroid perspectives (such as LV12, LV13]) in order to avoid confusions with the prime used in the notations for the cyclic parts (involving orientation-active elements and complementaries of flats of the dual) in the active decomposition of a single oriented matroid. Indeed, the present paper mixes the two settings, involving two single oriented matroids and the active decomposition of each of them (see details and references below Definition 11).

${ }^{3}$ This characterization is mentioned in [LV12], but not in the usual reference on oriented matroid theory [BLVS $\left.{ }^{+} 99\right]$. It is direct since vectors are signed subsets orthogonal to all cocircuits: a signed subset $C$ (meant to be a circuit of $M$ ) is not a vector of $N$ if and only if $C$ is not orthogonal to some cocircuit of $N$, that is if and only if it has a non-empty conformal intersection with some cocircuit of $N$.
} 
For such an oriented matroid perspective $M \rightarrow N$, the sets $O(M)$ and $O^{*}(N)$ will be respectively called, by extension of the terminology, the sets of active and dual-active elements of $M \rightarrow N$.

Now, we introduce a new construction, generalizing to oriented matroid perspectives a construction in oriented matroids detailed in Gio02, GLVa] (see also [GLV05, GLVb] in graphs). We refer the reader to these references for geometrical interpretations, that can readily be adapted in the setting of oriented matroid perspectives.

Let $M \rightarrow N$ be an oriented matroid perspective on a linearly ordered set $E$ with

$$
\begin{aligned}
O(M) & =\left\{g_{1}, \ldots, g_{\varepsilon}\right\}_{<}, \\
O^{*}(N) & =\left\{h_{1}, \ldots, h_{\iota}\right\}_{<} .
\end{aligned}
$$

We define $G_{\varepsilon}=\emptyset$ and, for $0 \leq k \leq \varepsilon-1$, we define

$$
G_{k}=\bigcup_{\substack{C \text { positive circuit of } M \\ \min (C) \geq g_{k+1}}} C .
$$

We define $H_{\iota}=E$ and, for $0 \leq k \leq \iota-1$, we define

$$
H_{k}=E \backslash \underset{\substack{C \text { positive cocircuit of } N \\ \min (C) \geq h_{k+1}}}{\bigcup} C .
$$

Observe that $G_{0}$ is the union of positive circuits of $M$ (i.e. the maximal positive vector of $M$ ), $E \backslash G_{0}$ is the union of positive cocircuits of $M$ (i.e. the maximal positive covector of $M$ ), $H_{0}$ is the union of positive circuits of $N$ (i.e. the maximal positive vector of $N$ ), and $E \backslash H_{0}$ is the union of positive cocircuits of $N$ (i.e. the maximal positive covector of $N$ ). Hence, by definition of an oriented matroid perspective, we have

$$
G_{0} \subseteq H_{0}
$$

Observe that we can have $G_{0}=H_{0}$, e.g. when $M=N$, and that we can also have $\emptyset=G_{0} \subset H_{0}=E$, when $M$ is acyclic and $N$ totally cyclic, as for the reorientations mentioned above counted by $t(M, N ; 0,0,1)$.

Definition 1. We call active filtration of $M \rightarrow N$ the sequence of nested subsets

$$
\emptyset=G_{\varepsilon} \subset \ldots \subset G_{1} \subset G_{0} \subseteq H_{0} \subset H_{1} \subset \ldots \subset H_{\iota}=E .
$$

And we call active partition of $M \rightarrow N$ the partition of $E$ given by

$$
E=\left(G_{\varepsilon-1} \backslash G_{\varepsilon}\right) \uplus \ldots \uplus\left(G_{0} \backslash G_{1}\right) \uplus\left(H_{0} \backslash G_{0}\right) \uplus\left(H_{1} \backslash H_{0}\right) \uplus \ldots \uplus\left(H_{\iota} \backslash H_{\iota-1}\right) .
$$

The parts contained in $G_{0}$ are called the cyclic parts, the parts contained in $E \backslash H_{0}$ are called the acyclic parts, and the part $H_{0} \backslash G_{0}$ (possibly empty) is called the hybrid part. We assume that the active filtration and active partition are always given along with the sets $G_{0}$ and $H_{0}$ (hence one can deduce the active filtration from the active partition and vice versa). 
The above definition generalizes active filtrations and active partitions of oriented matroids on a linearly ordered ground set, as defined in [Gio02, GLVa] (see also [GLV05, GLVb] in graphs). When $M=N$ the definitions coincide, and, in general, the acyclic parts for $M \rightarrow N$ equal the acyclic parts for $N$, and the cyclic parts for $M \rightarrow N$ equal the cyclic parts for $M$.

Moreover, note that we have, for $1 \leq k \leq \varepsilon$,

$$
\begin{aligned}
& g_{k}=\min \left(G_{k-1} \backslash G_{k}\right),
\end{aligned}
$$

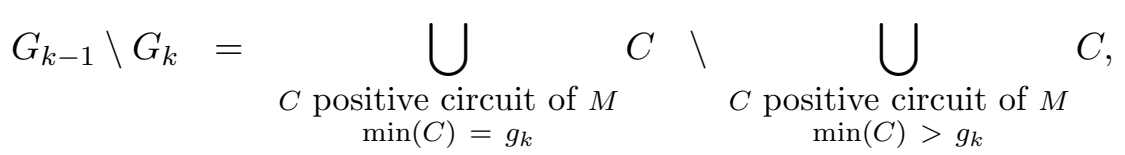

and, for $1 \leq k \leq \iota$

$$
\begin{aligned}
& h_{k}=\min \left(H_{k} \backslash H_{k-1}\right), \\
& H_{k} \backslash H_{k-1}=\underset{\substack{C \text { positive cocircuit of } N \\
\min (C)=h_{k}}}{\bigcup} C \backslash \underset{\substack{C \text { positive cocircuit of } N \\
\min (C)>h_{k}}}{\bigcup^{-}} C .
\end{aligned}
$$

Proposition 2. Let $M \rightarrow N$ be an oriented matroid perspective on a linearly ordered set E. Let $A \subseteq E$ be any union of parts of the active partition of $M \rightarrow N$. Then the oriented matroid perspective $-{ }_{A} M \rightarrow-{ }_{A} N$ has the same active partition as $M \rightarrow N$ (meaning precisely the same cyclic, acyclic and hybrid parts).

Proof. The proof is fairly simple, but let us take care to detail it. We will use the following notation, with the abuse of identifying a positive signed subset of $E$ with the subset of $E$ which is its support. For $e \in E, V(M ; e)$ denotes the union of all positive circuits of $M$ with smallest element greater than or equal to $e$. Equivalently, $V(M ; e)$ is the largest (for inclusion) positive vector with smallest element greater than or equal to $e$ (obtained by conformal composition of positive circuits).

First, we consider the case where $A=V(M ; a)$ for a given element $a \in O(M)$ (using the previous notations, it means that we have $a=g_{k+1}$ and $A=G_{k}$ for some $\left.0 \leq k \leq \varepsilon-1\right)$. Obviously, $A$ is also a positive vector of $-{ }_{A} M$. Let us fix $e \in E$ and let us prove that $V(M ; e)=V\left(-{ }_{A} M ; e\right)$.

Assume that $e \geq a$. Let $C$ be a positive circuit of $M$, with $\min (C) \geq e$. Then $C \subseteq A$ by definition of $A$, then $C$ is also a positive circuit of $-{ }_{A} M$. Conversely, let $C$ be a positive circuit of $-{ }_{A} M$, with $\min (C) \geq e$. Then the signed subset $A \circ C$ is a positive vector in $-{ }_{A} M$. Then, obviously, this signed subset $A \circ C$ is also a positive vector in $M$. This positive vector of $M$ has smallest element $a$, hence it is contained in $A$ by definition of $A$. So we have $C \subseteq A$, and so $C$ is also a positive circuit of $M$. Finally, when $e \geq a, M$ and $-{ }_{A} M$ have exactly the same positive circuits with smallest element greater than or equal to $e$, hence $V(M ; e)=V\left(-{ }_{A} M ; e\right)$.

Assume now that $e<a$. Let $C$ be a positive circuit of $M$, with $\min (C) \geq e$. Since $A$ is a positive vector of $-{ }_{A} M$ and $C$ is positive in $-{ }_{A} M$ on $C \backslash A$, we have that $A \circ C$ is a positive vector of $-{ }_{A} M$ with smallest element greater than or equal to $e$. This vector can be obtained in $-{ }_{A} M$ by a conformal composition of positive circuits, so $A \cup C$ is contained in $V\left(-{ }_{A} M ; e\right)$. So $V(M ; e) \subseteq V\left(-{ }_{A} M ; e\right)$. Conversely, let $C$ be a positive circuit of $-{ }_{A} M$, with $\min (C) \geq e$. By the same reasoning, we have that $A \circ C$ is a positive vector of $M$ with smallest element greater than or equal to $e$, that $A \cup C$ is contained in $V(M ; e)$, and that $V\left(-{ }_{A} M ; e\right) \subseteq V(M ; e)$.

Finally, we have proved that, for every $e \in E$, we have $V(M ; e)=V\left(-{ }_{A} M ; e\right)$. This implies that $O(M)=O\left(-{ }_{A} M\right)$ and, by definition of the active partition, that the cyclic parts of the active 
partition of $M \rightarrow N$ are equal to the cyclic parts of the active partition of $-{ }_{A} M \rightarrow-{ }_{A} N$ (precisley, using the previous notations, we have proved that, for $0 \leq k \leq \varepsilon-1$, the sets $G_{k}$ are the same when built in $M$ or in $\left.-{ }_{A} M\right)$.

Since $A$ is a union of positive circuits of $M$ and since $M \rightarrow N$ is an oriented perspective, we have that $A$ is a positive vector of $N$. Hence $A$ does not interfere with positive cocircuits of $N$, which remain exactly the same in $-{ }_{A} N$. Precisely, the acyclic parts of the active partition of $M \rightarrow N$ are obviously equal to the acyclic parts of the active partition of $-{ }_{A} M \rightarrow-{ }_{A} N$. And so the hybrid part also has to be the same in the active partitions of $M \rightarrow N$ and $-{ }_{A} M \rightarrow-{ }_{A} N$.

So we have proved the theorem in the case where $A=V(M ; a)$ for $a \in O(M)$. Using the previous notations for the active partition of $M \rightarrow N$, we have proved that $M \rightarrow N$ and $-{ }_{A} M \rightarrow-{ }_{A} N$ have the same active partitions when $A=G_{k}$ for any $0 \leq k \leq \varepsilon-1$ (hence for $\varepsilon$ possible non-empty sets $A$ ). Then, by transitivity, applying the result for $A=G_{k}$ and $A=G_{k+1}$, we have that $M \rightarrow N$ and $-{ }_{A} M \rightarrow-{ }_{A} N$ have the same active partitions when $A=G_{k} \backslash G_{k+1}$ for any $0 \leq k \leq \varepsilon-1$. Then, by transitivity again, we have proved the theorem when $A$ is any union of cyclic parts of the active partition of $M \rightarrow N$ (hence for $2^{\varepsilon}$ possible sets $A$ ).

Now, we can use the same reasoning to cocircuits in $N$ and acyclic parts of the active partition of $M \rightarrow N$, which is equivalent to apply the above result in the dual oriented matroid perspective $N^{*} \rightarrow M^{*}$. We obtain that the theorem is true when $A$ is any union of acyclic parts of the active partition of $M \rightarrow N$ (hence for $2^{\iota}$ possible sets $A$ ). By transtivity, combining the two dual results, we have that the theorem is true when $A$ is any union of cyclic or acyclic parts of the active partition of $M \rightarrow N$ (hence for $2^{\iota+\varepsilon}$ possible sets $A$ ).

Finally, it remains to handle the case where the hybrid part is non-empty. In this case, as the active partition of $M \rightarrow N$ is obviously the same as the active partition of $-{ }_{E} M \rightarrow-{ }_{E} N$, we have that the theorem is true when $A$ is any union of cyclic or acyclic parts of the active partition of $M \rightarrow N$, or its complementary (hence for $2^{\iota+\varepsilon+1}$ possible sets $A$ ). This means that $A$ can be any union of parts of the active partition of $M \rightarrow N$ (cyclic, acyclic, or hybrid).

Let $M \rightarrow N$ be an oriented matroid perspective on a linearly ordered set $E$. The set of all $-{ }_{A} M \rightarrow-{ }_{A} N$ for $A \subseteq E$ is called the set of reorientations of $M \rightarrow N$, being understood that this set is isomorphic to $2^{E}$. Note the slight abuse of notations, as we distinguish for instance between $-{ }_{A} M \rightarrow-{ }_{A} N$ and $-{ }_{E \backslash A} M \rightarrow-{ }_{E \backslash A} N$ as reorientations of $M \rightarrow N$, even though the two resulting oriented matroid perspectives are equal.

Definition 3. Let $M \rightarrow N$ be an oriented matroid perspective on a linearly ordered set $E$. We define the activity class of $M \rightarrow N$ as the set of its reorientations $-{ }_{A} M \rightarrow-{ }_{A} N$ where $A$ is any union of cyclic or acyclic parts of its active partition. Observe that we forbid reorienting the hybrid par 4. By Proposition 2 and extending this definition to all reorientations of $M \rightarrow N$, we define activity classes of reorientations of $M \rightarrow N$, that partition the set of reorientations of $M \rightarrow N$, and can be seen as classes of the equivalence relation defined by reorienting parts of the active partition.

\footnotetext{
${ }^{4}$ This is done in order to distinguish reorientations from their complementaries when the hybrid part is non-empty. This yields tighter classes, closer to Tutte polynomial properties, and meaningful notably in the proof of Theorem 7
} 
The above partition of the set of reorientations of $M \rightarrow N$ can be summed up as follows:

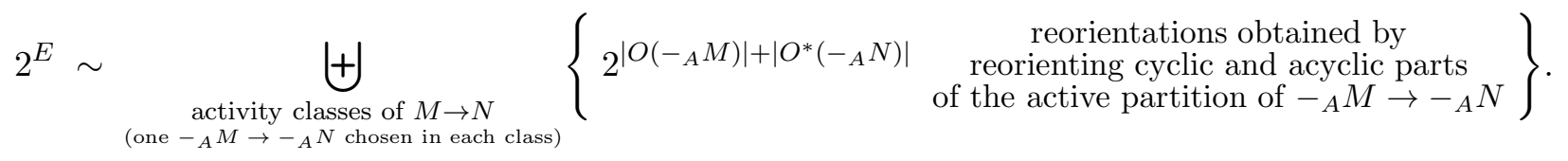

For practical purposes, it is also convenient to transpose this equivalence relation in terms of subsets $A \subset E$. In this setting, we can identify a reorientation $-{ }_{A} M \rightarrow-{ }_{A} N$ with the subset $A \subseteq E$ which defines it. Let us fix $A \subseteq E$. The active partition of $-{ }_{A} M \rightarrow-{ }_{A} N$ can be shortly denoted as:

$$
E=A_{0} \cup \underset{a \in O\left(-{ }_{A} M\right) \cup O^{*}\left(-{ }_{A} N\right)}{ } A_{a}
$$

where $A_{0}$ is the hybrid part, and where $A_{a}$ is either a cyclic or an acyclic part, with $a=\min \left(A_{a}\right)$ for $a \in O\left(-{ }_{A} M\right) \cup O^{*}\left(-{ }_{A} N\right)$. Then, the activity class of $-{ }_{A} M \rightarrow-{ }_{A} N$ can be renamed the activity class of $A \subseteq E$ w.r.t. $M \rightarrow N$ and denoted:

$$
c l_{M \rightarrow N}(A)=\left\{A \triangle\left(\biguplus_{a \in P \cup Q} A_{a}\right) \quad \mid P \subseteq O^{*}\left(-{ }_{A} N\right), Q \subseteq O\left(-{ }_{A} M\right)\right\} .
$$

Then, the different sets $c l_{M \rightarrow N}(A)$, for $A \in 2^{E}$, form a partition of $2^{E}$ :

$$
2^{E}=\underset{\left(\text { one } A \text { chosen in each } c l_{M \rightarrow N}(A)\right)}{c l_{M \rightarrow N}(A)}
$$

Observe that each $c l_{M \rightarrow N}(A)$ has a boolean lattice structure, yielding a partition of $2^{E}$ into boolean lattices. The above definition and construction generalize activivity classes of oriented matroids on a linearly ordered ground set, as defined in [Gio02, GLVa] (see also [GLV05, GLVb] in graphs).

Now, let us follow [LV12] and refine reorientation activities into four parameters, applied to reorientations of a given reference oriented matroid $M$.

Definition 4. Let $M$ be an oriented matroid on a linearly ordered set $E$ and $A \subseteq E$. We define:

$$
\begin{aligned}
\Theta_{M}(A) & =O\left(-{ }_{A} M\right) \backslash A, \\
\bar{\Theta}_{M}(A) & =O\left(-{ }_{A} M\right) \cap A, \\
\Theta_{M}^{*}(A) & =O^{*}\left(-{ }_{A} M\right) \backslash A, \\
\bar{\Theta}_{M}^{*}(A) & =O^{*}\left(-{ }_{A} M\right) \cap A .
\end{aligned}
$$

Hence we have $O\left(-{ }_{A} M\right)=\Theta_{M}(A) \uplus \bar{\Theta}_{M}(A)$ and (dually) $O^{*}\left(-{ }_{A} M\right)=\Theta_{M}^{*}(A) \uplus \bar{\Theta}_{M}^{*}(A)$.

Actually, in oriented matroids and oriented matroid perspectives as well, the parameters of Definition 4 situate any reorientation in its activity class seen as a boolean lattice. Let us detail this fundamental feature (in a similar way than done in [GLVb, GLVa]).

\footnotetext{
${ }^{5}$ The choice of letters $P$ and $Q$, in that order, respectively associated with dual-activity in $N$ and activity in $M$, might seem non-natural, but it is deliberate for the sake of matching with variables $x$ and $y$ respectively (and with internal and external subset activities respectively, as in Section 3 and in oriented matroids [GLVb, GLVa, LV13] ).
} 
Definition 5. Let $M$ be an oriented matroid on a linearly ordered set $E$ and $A \subseteq E$. The reorientation $-{ }_{A} M$ of $M$ is called active-fixed if no active element of $-{ }_{A} M$ is reoriented w.r.t. $M$, that is if $\bar{\Theta}_{M}(A)=O\left(-{ }_{A} M\right) \cap A=\emptyset$. It is called dual-active-fixed if no dual-active element of $-{ }_{A} M$ is reoriented w.r.t. $M$, that is if $\bar{\Theta}_{M}^{*}(A)=O^{*}\left(-{ }_{A} M\right) \cap A=\emptyset$.

For an oriented matroid perspective $M \rightarrow N$ on a linearly ordered set $E$ and $A \subseteq E$, by extension of the terminology, the reorientation $-{ }_{A} M \rightarrow-{ }_{A} N$ will be called active-fixed if $-{ }_{A} M$ is active-fixed (w.r.t. $M$ ) and dual-active-fixed if $-{ }_{A} N$ is dual active fixed (w.r.t. $\left.N\right)$.

Observation 6. Let $M \rightarrow N$ be an oriented matroid perspective on a linearly ordered set $E$. In each activity class of reorientations of $M \rightarrow N$, there is one and only one reorientation $-{ }_{A} M \rightarrow-{ }_{A} N$ which is active-fixed and dual-active-fixed (that is such that all active elements of $M$ and dual-active elements of $N$ are not reoriented with respect to the initial $M \rightarrow N$ ).

In other words, continuing the above setting, in the activity class $c l_{M \rightarrow N}(A)$, one can choose as representative the subset $A$ such that

$$
\bar{\Theta}_{M}(A)=O\left(-{ }_{A} M\right) \cap A=\emptyset \quad \text { and } \quad \bar{\Theta}_{N}^{*}(A)=O^{*}\left(-{ }_{A} N\right) \cap A=\emptyset .
$$

So, for this special $A$ and according to the above definition of $c l_{M \rightarrow N}(A)$, an element $A^{\prime}$ of $c l_{M \rightarrow N}(A)$ is situated in $c l_{M \rightarrow N}(A)$ by $P \subseteq O^{*}\left(-{ }_{A} N\right)=O^{*}\left(-A_{A^{\prime}} N\right)$ and $Q \subseteq O\left(-{ }_{A} M\right)=O\left(-A_{A^{\prime}} M\right)$ such that:

$$
\begin{aligned}
\Theta_{M}\left(A^{\prime}\right) & =O\left(-{ }_{A} M\right) \backslash Q, \\
\bar{\Theta}_{M}\left(A^{\prime}\right) & =Q, \\
\Theta_{N}^{*}\left(A^{\prime}\right) & =O^{*}\left(-{ }_{A} N\right) \backslash P, \\
\bar{\Theta}_{N}^{*}\left(A^{\prime}\right) & =P .
\end{aligned}
$$

Now, we get the following Theorem 7 , a four variable expression of the Tutte polynomial in terms of the above four parameters, stated and proved by deletion/contraction in [LV12]. Here, we give a short natural proof of this theorem, using the Tutte polynomial formula in terms of orientation activities, the partition of the set of reorientations into activity classes, and their boolean lattice structure as discussed above.

Theorem 7 ([LV12]). Let $M \rightarrow N$ be an oriented matroid perspective on a linearly ordered set $E$. We have

$$
t(M, N ; x+u, y+v, 1)=\sum_{A \subseteq E} x^{\left|\Theta_{N}^{*}(A)\right|} u^{\left|\bar{\Theta}_{N}^{*}(A)\right|} y^{\left|\Theta_{M}(A)\right|} v^{\left|\bar{\Theta}_{M}(A)\right|} .
$$

Proof. Let us denote $[E x p]=\sum_{A \subseteq E} x^{\left|\Theta_{N}^{*}(A)\right|} u^{\left|\bar{\Theta}_{N}^{*}(A)\right|} y^{\left|\Theta_{M}(A)\right|} v^{\left|\bar{\Theta}_{M}(A)\right|}$. Since $2^{E}$ is partitioned into activity classes by Definition [3, and denoting $\operatorname{cl}(A)$ for short instead of $c_{M \rightarrow N}(A)$, we have:

$$
[E x p]=\sum_{\begin{array}{c}
c l(A) \\
\text { one } A \text { for each class }
\end{array}} \sum_{A^{\prime} \in \operatorname{cl}(A)} x^{\left|\Theta_{N}^{*}\left(A^{\prime}\right)\right|} u^{\left|\bar{\Theta}_{N}^{*}\left(A^{\prime}\right)\right|} y^{\left|\Theta_{M}\left(A^{\prime}\right)\right|} v^{\left|\bar{\Theta}_{M}\left(A^{\prime}\right)\right|}
$$

As discussed above, one can choose a representative $A$ of $\operatorname{cl}(A)$ such that $O\left(-{ }_{A} M\right) \cap A=\emptyset$ and $O^{*}\left(-{ }_{A} N\right) \cap A=\emptyset$, and use the structure of $\operatorname{cl}(A)$, to reformulate the expression as follows:

$$
[E x p]=\sum_{\substack{c l(A) \\ \text { one } A \text { for each class }}} \sum_{\substack{P \subseteq O^{*}\left(-{ }_{A} N\right) \\ Q \subseteq O\left(-{ }_{A} M\right)}} x^{\left|O^{*}\left(-{ }_{A} N\right) \backslash P\right|} u^{|P|} y^{\left|O\left(-{ }_{A} M\right) \backslash Q\right|} v^{|Q|}
$$


Now we can simply rewrite this expression as follows and use the binomial formula:

$$
\begin{aligned}
{[E x p] } & =\sum_{\substack{c l(A) \\
\text { one for each class }}}\left(\sum_{P \subseteq O^{*}\left(-{ }_{A} N\right)} x^{\left|O^{*}\left(-{ }_{A} N\right) \backslash P\right|} u^{|P|}\right)\left(\sum_{Q \subseteq O\left(-{ }_{A} M\right)} y^{\left|O\left(-{ }_{A} M\right) \backslash Q\right|} v^{|Q|}\right) \\
& =\sum_{\substack{c l(A) \\
\text { one } A \text { for each class }}}(x+u)^{\left|O^{*}\left(-{ }_{A} N\right)\right|}(y+v)^{\left|O\left(-{ }_{A} M\right)\right|}
\end{aligned}
$$

For a better readability, let us denote $o\left(-{ }_{A} M\right)$ for $\left|O\left(-{ }_{A} M\right)\right|$, and $o^{*}\left(-{ }_{A} M\right)$ for $\left|O^{*}\left(-{ }_{A} M\right)\right|$. Since the activity class $c l(A)$ has $2^{o\left(-{ }_{A} M\right)+o^{*}\left(-{ }_{A} N\right)}$ elements with the same activities, we have:

$$
\begin{aligned}
{[E x p] } & =\sum_{\substack{c l(A) \\
\text { one } A \text { for each class }}} \frac{1}{2^{o^{*}\left(-{ }_{A} N\right)+o\left(-{ }_{A} M\right)}} \sum_{A^{\prime} \in c l(A)}(x+u)^{o^{*}\left(-{ }_{A^{\prime}} N\right)}(y+v)^{o\left(-{ }_{A^{\prime}} M\right)} \\
& =\sum_{\substack{c l(A) \\
\text { one } A \text { for each class }}} \sum_{A^{\prime} \in \operatorname{cl}(A)}\left(\frac{x+u}{2}\right)^{o^{*}\left(-{ }_{A^{\prime}} N\right)}\left(\frac{y+v}{2}\right)^{o\left(-{ }_{A^{\prime}} M\right)} \\
& =\sum_{A \subseteq E}\left(\frac{x+u}{2}\right)^{o^{*}\left(-{ }_{A} N\right)}\left(\frac{y+v}{2}\right)^{o\left(-{ }_{A} M\right)} \\
& =t(M, N ; x+u, y+v, 1)
\end{aligned}
$$

by the formula in terms of orientation activities from [LV84] recalled earlier.

Numerous Tutte polynomial formulas, for oriented matroid perspectives or for oriented matroids as well, can be directly obtained from Theorem 7 , for instance by replacing variables $(x, u, y, v)$ with $(x / 2, x / 2, y / 2, y / 2)$, or $(x+1,-1, y+1,-1)$, or $(2,0,0,0) \ldots$ Let us give examples of such formulas in the corollary below. These formulas, and a detailed example for this set of formulas, are given [LV12]. Actually, a total of 25 expansions, 9 different up to reordering, could be thus obtained (in a similar way than in [GT90, LV13] for formulas from subset activities).

Corollary 8 ([LV12] $)$. Let us denote $\theta_{M}(A)$ instead of $\left|\Theta_{M}(A)\right|$, etc. Let $p, q$ be two non-negative integers. Let $M \rightarrow N$ be an oriented matroid perspective on a linearly ordered set $E$. We have (among possible variants as discussed above):

$$
\begin{aligned}
& t(M, N ; x, y, 1)=\sum_{A \subseteq E}(x-1)^{\theta_{N}^{*}(A)}(y-1)^{\theta_{M}(A)}=\sum_{\substack{A \subseteq E \\
\bar{\theta}_{N}^{*}(A)=0 \\
\bar{\theta}_{M}(A)=0}} x^{\theta_{N}^{*}(A)} y^{\theta_{M}(A)} \\
& t(M, N ; 0,0,1)=\sum_{A \subseteq E}(-1)^{\theta_{N}^{*}(A)+\theta_{M}(A)} \\
& \frac{\partial^{p+q} t}{\partial x^{p} \partial y^{q}}(M, N ; x, y, 1)=p ! q ! \sum_{\substack{A \subseteq E \\
\bar{\theta}_{N}^{*}(A)=p \\
\bar{\theta}_{M}(A)=q}} x^{\theta_{N}^{*}(A)} y^{\theta_{M}(A)} .
\end{aligned}
$$

Let $M$ be an oriented matroid on a linearly ordered set $E$. We have: 


$$
\begin{aligned}
& t(M ; x+u, y+v)=\sum_{A \subseteq E} x^{\theta_{M}^{*}(A)} u^{\bar{\theta}_{M}^{*}(A)} y^{\theta_{M}(A)} v^{\bar{\theta}_{M}(A)} \\
& t(M ; x, y)=\sum_{A \subseteq E}(x-1)^{\theta_{M}^{*}(A)}(y-1)^{\theta_{M}(A)}=\sum_{\substack{A \subseteq E \\
\bar{\theta}_{M}^{*}(A)=0 \\
\bar{\theta}_{M}(A)=0}} x^{\theta_{M}^{*}(A)} y^{\theta_{M}(A)} \\
& t(M ; 2,0)=\sum_{\substack{A \subseteq E \\
\bar{\theta}_{M}^{*}(A)=\theta_{M}(A)=\bar{\theta}_{M}(A)=0}} 2^{\theta_{M}^{*}(A)}=\sum_{\substack{A \subseteq E \\
\theta_{M}^{*}(A)=\theta_{M}(A)=\bar{\theta}_{M}(A)=0}} 2^{\bar{\theta}_{M}^{*}(A)} \\
& \frac{\partial^{p+q}}{\partial x^{p} \partial y^{q}} t(M ; x, y)=p ! q ! \sum_{\substack{A \subseteq E \\
\bar{\theta}_{M}^{*}(A)=p \\
\bar{\theta}_{M}(A)=q}} x^{\theta_{M}^{*}(A)} y^{\theta_{M}(A)} .
\end{aligned}
$$

Proof. Direct by Theorem 17, use Taylor's theorem for the derivative expressions.

Observation 9. We have $\bar{\theta}_{N}^{*}(A)=p$ and $\bar{\theta}_{M}(A)=q$ if $q$ active elements of $-{ }_{A} M$ and $p$ dualactive elements of $-{ }_{A} N$ are reoriented in $-{ }_{A} M \rightarrow-{ }_{A} N$ w.r.t. $M \rightarrow N$ (or equally w.r.t. the unique reorientation of $M \rightarrow N$ in the same activity class as $-{ }_{A} M \rightarrow-{ }_{A} N$ which is active-fixed and dual-active-fixed).

Let us end with a proposition summing up how the Tutte polynomial coefficients and evaluations count sets of reorientations based on activity classes and their representatives. It is direct from the previous definitions and results. Let us mention that these reorientations are addressed in oriented matroids and bijectively related to certain subsets in terms of subset activities in [GLVb, GLVa].

Proposition 10. Let $M \rightarrow N$ be an oriented matroid perspective on a linearly ordered set $E$. Let us denote $t_{i, j}$ the coefficient of $x^{i} y^{j}$ in $t(M, N ; x, y, 1)$.

Then $t_{i, j}$ equals the number of activity classes of reorientations of $M \rightarrow N$ with $i$ dual-active elements and $j$ active elements. Equally (by Observation 6), $t_{i, j}$ equals the number of reorientations of $M \rightarrow N$, with $i$ dual-active elements and $j$ active elements, which are active-fixed (w.r.t. $M$ ) and dual-active fixed (w.r.t. $N$ ).

Furthermore, by combining these counting results, certain remarkable subsets of reorientations are enumerated by Tutte polynomial evaluations as shown in Table 2. In particular, $t(M, N ; 1,1,1)$ counts the total number of activity classes of reorientations. 


\begin{tabular}{|l|c|}
\hline $\begin{array}{l}\text { reorientations }-{ }_{A} M \rightarrow-{ }_{A} N \text { with } \\
\text { (resp. activity classes with) }\end{array}$ & are counted by \\
\hline no condition & $t(M, N ; 2,2,1)$ \\
acyclic $-{ }_{A} M$ & $t(M, N ; 2,0,1)$ \\
totally cyclic $-{ }_{A} N$ & $t(M, N ; 0,2,1)$ \\
acyclic $-{ }_{A} M$ and dual-active-fixed $-{ }_{A} N$ & $t(M, N ; 1,0,1)$ \\
$\quad$ (resp. acyclic $\left.-{ }_{A} M\right)$ & $t(M, N ; 0,1,1)$ \\
active-fixed $-{ }_{A} M$ and totally cyclic $-{ }_{A} N$ & $t(M, N ; 2,1,1)$ \\
$\quad$ (resp. totally cyclic $\left.-{ }_{A} N\right)$ & $t(M, N ; 1,2,1)$ \\
active-fixed $-{ }_{A} M$ & $t(M, N ; 1,1,1)$ \\
dual-active-fixed $-{ }_{A} N$ & \\
active-fixed $-{ }_{A} M$ and dual-active-fixed $-{ }_{A} N$ & \\
$\quad$ (resp. $n o$ condition) & $t(M, N ; 0,0,1)$ \\
acyclic $-{ }_{A} M$ and totally cyclic $-{ }_{A} N$ & (resp. acyclic $-{ }_{A} M$ and totally cyclic $\left.-{ }_{A} N\right)$
\end{tabular}

Table 1: Enumeration of certain reorientations based on representatives of activity classes (Proposition 10).

\section{Building on subset activities in matroid perspectives}

As explained in the introduction, this section sums up and completes the construction from [LV13] of the Tutte polynomial of a matroid perspective in terms of subset activities. We highlight the close relation between a structural partition and an enumerative Tutte polynomial expansion. Actually, this relation is remarkable in matroids as well. The reader is referred to [Oxl11] for background on matroids, and more specifically to [Kun86] or [Oxl11, Section 7.3] on perspectives of matroids. Let $M$ and $N$ be two matroids on the set $E$. The ordered pair $(M, N)$ constitutes a (matroid) perspective (also called quotient map, or strong map up to unimportant variants), and is denoted $M \rightarrow N$, if the following equivalent properties are satisfied:

- $r_{N}(X)-r_{N}(Y) \leq r_{M}(X)-r_{M}(Y)$ for all $Y \subseteq X \subseteq E$;

- every flat of $N$ is a flat of $M$;

- every cocircuit of $N$ is a union of cocircuits of $M$;

- every circuit of $M$ is a union of circuits of $N$;

- no circuit of $M$ and cocircuit of $N$ intersect in exactly one element6;

- there is a matroid $N$ on $E \uplus A$ such that $M=N \backslash A$ and $N=N / A$.

\footnotetext{
${ }^{6}$ This characterization is mentioned in [LV13, but not in the usual references on matroid theory [Kun86, Oxl11]. We have not found a proof in the literature. Let us give one here. Assume a circuit $C$ of $M$ and a cocircuit $D$ of $N$ satisfy $C \cap D=\{e\}$. Then, $E \backslash D$ is a flat of $N$ which is not a flat of $M$. Indeed, $E \backslash D$ is a flat of $N$ as the complement of a cocircuit, and $E \backslash D$ is not a flat of $M$, otherwise $C \backslash\{e\} \subseteq E \backslash D$ would imply $e \in E \backslash D$. Conversely, assume $F$ is a flat of $N$ which is not a flat of $M$. Then, there exists a circuit $C$ of $M$ and $e \in C$ such that $e \notin F$ and $C \backslash\{e\} \subseteq F$. Since $F$ is a flat of $N$, let us consider a basis $B$ of $N$ formed by a basis of $N(F)$, plus $\{e\}$, plus some elements of $E$. Then, the closure of $B \backslash\{e\}$ in $N$ contains $F$, and its complement is a cocircuit $D$ of $N$. By construction we have $C \backslash\{e\} \subseteq F \subseteq E \backslash D$ and $e \in D$, hence finally $C \cap D=\{e\}$.
} 
Observe that, for a matroid $M, M \rightarrow M$ is a perspective, meaning that every result given for perspectives is also true for matroids by setting $M=N$. Actually, if $M \rightarrow N$ is a matroid perspective and $r(M)=r(N)$ then $M=N$. Let us observe that if $M \rightarrow N$ is a matroid perspective then $N^{*} \rightarrow M^{*}$ is also a matroid perspective.

Let $M$ be a matroid on a linearly ordered set $E$. For $A \subseteq E$, we define:

$$
\begin{aligned}
\operatorname{Int}_{M}(A) & =\{e \in A \mid e=\min (C), C \text { cocircuit contained in }(E \backslash A) \cup\{e\}\}, \\
\operatorname{Ext}_{M}(A) & =\{e \in E \backslash A \mid e=\min (C), C \text { circuit contained in } A \cup\{e\}\} .
\end{aligned}
$$

Then the parameters $\left|\operatorname{Int}_{M}(A)\right|$ and $\left|\operatorname{Ext}_{M}(A)\right|$ are respectively called the internal activity and external activity of $A$ in $M$. If $A$ is a basis of $M$, then the above definitions of $\operatorname{Int}_{M}(A), \iota_{M}(A)$, $\operatorname{Ext}_{M}(A)$, and $\varepsilon_{M}(A)$ are the same as the usual ones for basis activities. Extending the usual notion of activities from matroid basis to subsets enables the following expression of the Tutte polynomial of a matroid perspective in terms of independent/spanning subset activities from [LV80, LV99], which extends the usual one of a matroid in terms of basis activities (the initial definition of the Tutte polynomial of a matroid perspective in terms of rank functions is recalled in Section (1).

Let $M \rightarrow N$ be a matroid perspective on a linearly ordered set $E$. By [LV80, LV99], its Tutte polynomial satisfies

$$
t(M, N ; x, y, z)=\sum_{\substack{B \subseteq E \\ B \text { independant in } M \\ \text { and spanning in } N}} x^{\left|\operatorname{Int}_{N}(B)\right|} y^{\left|\operatorname{Ext}_{M}(B)\right|} z^{r(M)-r(N)-\left(r_{M}(B)-r_{N}(B)\right)} .
$$

Beyond this, subset activities yield the following remarkable partition into boolean intervals of subsets, as shown in [LV13] by applying the general construction of [Daw81]. We have

$$
2^{E}=\biguplus_{\substack{B \subseteq E \\ B \text { independent in } M \\ \text { and spanning in } N}}\left[B \backslash \operatorname{Int}_{N}(B), B \cup \operatorname{Ext}_{M}(B)\right] .
$$

Now, let $M$ be a matroid on a linearly ordered set $E$, for $A \subseteq E$, we define:

$$
\begin{aligned}
P_{M}(A) & =\{e \in E \backslash A \mid e=\min (C), C \text { cocircuit contained in } E \backslash A\}, \\
Q_{M}(A) & =\{e \in A \mid e=\min (C), C \text { circuit contained in } A\} .
\end{aligned}
$$

The cardinalities of these two sets turn out to be well-known, and independent of the ordering of $E$ :

$$
\begin{aligned}
\left|P_{M}(A)\right| & =r(M)-r_{M}(A), \\
\left|Q_{M}(A)\right| & =|A|-r_{M}(A) .
\end{aligned}
$$

Finally, subset activities can be seen as parameters that situate the position of a subset inside the boolean interval which contains it with respect to the above partition. Precisely, let $B \subseteq E$ be independent in $M$ and spanning in $N$, and let $A$ be in the interval $\left[B \backslash \operatorname{Int}_{N}(B), B \cup \operatorname{Ext}_{M}(B)\right]$. We have the following relations, showing the consistency of the whole above setting:

$$
\operatorname{Int}_{N}(A)=\operatorname{Int}_{N}(B) \cap A,
$$




$$
\begin{aligned}
P_{N}(A) & =\operatorname{Int}_{N}(B) \backslash A, \\
\operatorname{Ext}_{M}(A) & =\operatorname{Ext}_{M}(B) \backslash A, \\
Q_{M}(A) & =\operatorname{Ext}_{M}(B) \cap A .
\end{aligned}
$$

More properties of the above partition into intervals, and, more specifically, of the relations between an independent/spanning subset and the subsets in its associated interval, are listed in LV13, Prop. 4.4]. However, one non-trivial property is missing in this list and never mentioned in [LV13], whereas it is required to prove the main theorem 7 . The next proposition fills this gap, and it is also required in our proof of the main theorem below.

Proposition 11. Let $M \rightarrow N$ be a matroid perspective on a linearly ordered set $E$. Let $B \subseteq E$ be independent in $M$ and spanning in $N$, and let $A \in\left[B \backslash \operatorname{Int}_{N}(B), B \cup \operatorname{Ext}_{M}(B)\right]$. Then we have

$$
r_{M}(A)-r_{N}(A)=r_{M}(B)-r_{N}(B) \text {. }
$$

Proof. We have $A=B \backslash P \cup Q$ with $P \subseteq \operatorname{Int}_{N}(B) \subseteq B$ and $Q \subseteq \operatorname{Ext}_{M}(B) \subseteq E \backslash B$. First, let us consider any $e \in Q$ and $f \in P$. By definition of $\operatorname{Ext}_{M}(B)$, there exists a circuit $C$ of $M$ with $C \backslash\{e\} \subseteq B$ and $e=\min (C)$. By definition of $\operatorname{Int}_{N}(B)$, there exists a cocircuit $D$ of $N$ with $D \backslash\{f\} \subseteq E \backslash B$ and $f=\min (D)$. By construction, we have $C \cap D \subseteq\{e, f\}$. Let us prove that $C \cap D=\emptyset$. Otherwise, by properties of a matroid perspective (no circuit of $M$ and cocircuit of $N$ intersect in exactly one element), we must have $e \neq f$ and $C \cap D=\{e, f\}$. In this case, we have $e \leq f$ by properties of $e$, and $f \leq e$ by properties of $f$, hence $e=f$, a contradiction. So $C \cap D=\emptyset$. This conclusion is true for any choice of $e$ and $f$, so we have in addition $C \cap P=\emptyset$ and $D \cap Q=\emptyset$.

Now, let us place in $M$. Since $B$ is independent in $M$, we have $r_{M}(B)=|B|$. Moreover, we have that $B \backslash P$ is independent in $M$. For $e \in Q$ and a circuit $C$ of $M$ as above, we have shown that $C \cap P=\emptyset$, hence $C \backslash\{e\} \subseteq B \backslash P$, hence $e$ belongs to the closure of $B \backslash P$ in $M$. This is true for any $e \in Q$, so $A=B \backslash P \cup Q$ is contained in the closure of $B \backslash P$ in $M$. So we have $r_{M}(A)=r_{M}(B \backslash P)$. Since $P \subseteq B$ and $B \backslash P$ is independent, we finally have $r_{M}(A)=|B|-|P|$.

Now, let us place in $N$. Since $B$ is spanning in $N$, we have $r_{N}(B)=r(N)$. For $f \in P$ and a cocircuit $D$ of $N$ as above, we have $D \backslash\{f\} \subseteq E \backslash B$, hence $B \backslash\{f\}$ spans the hyperplane $E \backslash D$ of $N$ (flat with rank $r(N)-1$ ). Moreover, we have shown that $D \cap Q=\emptyset$, that is: $Q$ is contained in this hyperplane $E \backslash D$ of $N$. All this is true for any $f \in P$, so let us consider the intersection of the associated hyperplanes $E \backslash D$ when $f$ ranges $P$. We get that the flat spanned by $B \backslash P$ has rank $r(N)-|P|$ and that $Q$ is contained in this flat. So we have shown that $r_{N}(A)=r(N)-|P|$.

Finally, we have $r_{M}(A)-r_{N}(A)=|B|-|P|-(r(N)-|P|)=|B|-r(N)=r_{M}(B)-r_{N}(B)$.

Now, let us consider the main theorem below. This general Tutte polynomial expansion formula, known for matroids from [GT90] and generalized to matroid perspectives in [LV13], contains a lot of formulas by suitable variable choices, see [GT90, LV13]. Notably, a charm of this formula is that it contains the two formulas in three variable previously given: the first (in terms of rank function) by using the variables $(1, x-1,1, y-1, z)$, the second (in terms of activities for independent/spanning subsets) by using the variables $(x, 0, y, 0, z)$. We give below a short natural proof,

\footnotetext{
${ }^{7}$ Precisely: [LV13, Lemma 4.6] actually follows from [LV13, Prop. 4.4] as written in [LV13] but only once the argument of Proposition 11 is established, ensuring that, in the statement of [LV13, Lemma 4.6], we have $\operatorname{rcd}_{M, M^{\prime}}(A)=\operatorname{rcd}_{M, M^{\prime}}(B)$, so that the parameter $k$ makes sense and is correctly handled, .
} 
highlighting how this formula is the enumerative counterpart of the fundamental structural partition into intervals addressed above. The proof in [LV13] is in terms of expressions of derivatives of the Tutte polynomial, which is indirectly equivalent by means of Taylor's theorem, but which is not combinatorially striking the same way.

Theorem 12 ([LV13] $)$. Let $M \rightarrow N$ be a matroid perspective on a linearly ordered set $E$. We have

$$
t(M, N ; x+u, y+v, z)=\sum_{A \subseteq E} x^{\left|\operatorname{Int}_{N}(A)\right|} u^{\left|P_{N}(A)\right|} y^{\left|\operatorname{Ext}_{M}(A)\right|} v^{\left|Q_{M}(A)\right|} z^{r(M)-r(N)-\left(r_{M}(A)-r_{N}(A)\right)}
$$

Proof. For $A \subseteq E$, let us denote $\operatorname{rcd}_{M, N}(A)=r(M)-r(N)-\left(r_{M}(A)-r_{N}(A)\right)$. By the expression of $t(M ; x+u, y+v)$ recalled above, we have

$$
t(M, N ; x+u, y+v, z)=\sum_{\substack{B \subseteq E \\ B \text { independant in } M \\ \text { and spanning in } N}}(x+u)^{\left|\operatorname{Int}_{N}(B)\right|}(y+v)^{\left|\operatorname{Ext}_{M}(B)\right|} z^{r c d_{M, N}(B)} .
$$

By the binomial formula, this expression equals

$$
\sum_{\substack{B \subseteq E \\ B \text { independant in } M \\ \text { and spanning in } N}}\left(\sum_{A^{\prime} \subseteq \operatorname{Int}_{N}(B)} x^{\left|A^{\prime}\right|} u^{\left|\operatorname{Int}_{N}(B) \backslash A^{\prime}\right|}\right)\left(\sum_{A^{\prime \prime} \subseteq \operatorname{Ext}_{M}(B)} y^{\left|\operatorname{Ext}_{M}(B) \backslash A^{\prime \prime}\right|} v^{\left|A^{\prime \prime}\right|}\right) z^{r c d_{M, N}(B)} .
$$

Since $\operatorname{Int}_{N}(B) \subseteq B$ and $\operatorname{Ext}_{M}(B) \subseteq E \backslash B$, we have $\operatorname{Int}_{N}(B) \cap \operatorname{Ext}_{M}(B)=\emptyset$. So, one has a bijection between pairs $\left(A^{\prime}, A^{\prime \prime}\right)$ involved in the above expression and subsets $A=A^{\prime} \uplus A^{\prime \prime}$ of $\operatorname{Int}_{N}(B) \uplus \operatorname{Ext}_{M}(B)$, hence this expression equals

$$
\sum_{\substack{B \subseteq E \\ B \text { independant in } M \\ \text { and spanning in } N}}\left(\sum_{A \subseteq \operatorname{Int}_{N}(B) \uplus \operatorname{Ext}_{M}(B)} x^{\left|\operatorname{Int}_{N}(B) \cap A\right|} u^{\left|\operatorname{Int}_{N}(B) \backslash A\right|} y^{\left|\operatorname{Ext}_{M}(B) \backslash A\right|} v^{\left|\operatorname{Ext}_{M}(B) \cap A\right|}\right) z^{r c d_{M, N}(B)} .
$$

Since $\left(B \backslash \operatorname{Int}_{N}(B)\right) \cap\left(\operatorname{Int}_{N}(B) \uplus \operatorname{Ext}_{M}(B)\right)=\emptyset$, the mapping $A \mapsto A \cup\left(B \backslash \operatorname{Int}_{N}(B)\right)$ yields an isomorphism between the two boolean intervals $\left[\emptyset, \operatorname{Int}_{N}(B) \uplus \operatorname{Ext}_{M}(B)\right]$ and $\left[B \backslash \operatorname{Int}_{N}(B), B \cup\right.$ $\left.\operatorname{Ext}_{M}(B)\right]$, which does not change the sets $\operatorname{Int}_{N}(B) \cap A, \operatorname{Int}_{N}(B) \backslash A, \operatorname{Ext}_{M}(B) \backslash A$, and $\operatorname{Ext}_{M}(B) \cap A$. So the above expression can be equivalently written

$$
\sum_{\substack{B \subseteq E \\ B \text { independant in } M \\ \text { and spanning in } N}}\left(\sum_{\substack{A \in\left[B \backslash \operatorname{Int}_{N}(B), B \cup \operatorname{Ext}_{M}(B)\right]}} x^{\left|\operatorname{Int}_{N}(B) \cap A\right|} u^{\left|\operatorname{Int}_{N}(B) \backslash A\right|} y^{\left|\operatorname{Ext}_{M}(B) \backslash A\right|} v^{\left|\operatorname{Ext}_{M}(B) \cap A\right|}\right) z^{r c d_{M, N}(B)} .
$$

By Proposition 11, we have $\operatorname{rcd}_{M, N}(B)=\operatorname{rcd}_{M, N}(A)$ for $B$ independent in $M$ and spanning in $N$ and for $A \in\left[B \backslash \operatorname{Int}_{N}(B), B \cup \operatorname{Ext}_{M}(B)\right]$. Hence the above expression equals

$$
\sum_{\substack{B \subseteq E \\
\begin{array}{c}
B \text { independant in } M \\
\text { and spanning in } N
\end{array}}} \sum_{\substack{A \in\left[B \backslash \operatorname{Int}_{N}(B), B \cup \operatorname{Ext}_{M}(B)\right]}} x^{\left|\operatorname{Int}_{N}(B) \cap A\right|} u^{\left|\operatorname{Int}_{N}(B) \backslash A\right|} y^{\left|\operatorname{Ext}_{M}(B) \backslash A\right|} v^{\left|\operatorname{Ext}_{M}(B) \cap A\right|} z^{r c d_{M, N}(A)} .
$$

Since $2^{E}$ is the disjoint union of intervals $\left[B \backslash \operatorname{Int}_{M}(B), B \cup \operatorname{Ext}_{M}(B)\right]$ for $B$ independent in $M$ and spanning in $N$ (as recalled above), this expression equals 
$\sum_{A \subseteq E} x^{\left|\operatorname{Int}_{N}(B) \cap A\right|} u^{\left|\operatorname{Int}_{N}(B) \backslash A\right|} y^{\left|\operatorname{Ext}_{M}(B) \backslash A\right|} v^{\left|\operatorname{Ext}_{M}(B) \cap A\right|} z^{r c d_{M, N}(A)}$.

Finally, replacing the exponents as recalled above, this expression equals the required one.

\section{References}

[BGdON09] J.M. Brunat, A. Guedes de Oliveira, and M. Noy. Partitions of a finite boolean lattice into intervals. European J. Combin., 30:1801-1809, 2009.

[Bjö92] A. Björner. Homology an shellability of matroids and geometric lattices. In N. White, editor, Matroid applications, volume 40 of Encyclopedia of Mathematics and Its Applications. Cambridge University Press, 1992.

[BLVS $\left.{ }^{+} 99\right]$ A. Björner, M. Las Vergnas, B. Sturmfels, N. White, and G. Ziegler. Oriented Matroids, volume 46 of Encyclopedia of Mathematics and Its Applications. Cambridge University Press, second edition, 1999.

[Cra69] H.H. Crapo. The tutte polynomial. Aequationes Mathematicae, 3:211-229, 1969.

[Daw81] J.E. Dawson. A construction for a family of sets and its application to matroids. In Springer, editor, Lect. Notes in Math. (Comb. Math. VIII, Gelong, 1980), volume 884, pages 136-147, 1981.

[Gioa] E. Gioan. The Tutte polynomial of matroid perspectives. In J. Ellis-Monaghan and I. Moffatt, editors, Handbook of the Tutte Polynomial, CRC Monographs and Research Notes in Mathematics. Submitted.

[Giob] E. Gioan. The Tutte polynomial of oriented matroids. In J. Ellis-Monaghan and I. Moffatt, editors, Handbook of the Tutte Polynomial, CRC Monographs and Research Notes in Mathematics. Submitted.

[Gio02] E. Gioan. Correspondance naturelle entre bases et réorientations des matroïdes orientés. $\mathrm{PhD}$ thesis, University of Bordeaux 1, 2002. (available at http://www.lirmm.fr/ gioan).

[GLVa] E. Gioan and M. Las Vergnas. The active bijection - 2.b - Decomposition of activities for oriented matroids, and general definitions of the active bijection. Submitted, preprint available at arXiv:180\%.065\%8.

[GLVb] E. Gioan and M. Las Vergnas. The active bijection for graphs. Submitted, preprint available at arXiv:1807.06545.

[GLV05] E. Gioan and M. Las Vergnas. Activity preserving bijections between spanning trees and orientations in graphs. Discrete Mathematics, 298:169-188, 2005.

[GLV07] E. Gioan and M. Las Vergnas. Fully optimal bases and the active bijection in graphs, hyperplane arrangements, and oriented matroids. Electronic Notes in Discrete Mathematics, 29:365-371, 2007. Proceedings EuroComb 2007 (Sevilla).

[GM97] G. Gordon and E. McMahon. Interval partitions and activities for the greedoid tutte polynomial. Adv. Appl. Math., 18:33-49, 1997.

[GT90] G. Gordon and L. Traldi. Generalized activities and the Tutte polynomial. Disc. Math., 85:167176, 1990.

[Kun86] J. Kung. Strong maps. In N. White, editor, Theory of matroids, volume 26 of Encyclopedia of Mathematics and Its Applications. Cambridge University Press, 1986.

[LV80] M. Las Vergnas. On the Tutte polynomial of a morphism of matroids. Annals of Discrete Mathematics, 8:7-20, 1980.

[LV84] M. Las Vergnas. The Tutte polynomial of a morphism of matroids II. Activities of orientations. In J.A. Bondy and U.S.R. Murty, editors, Progress in Graph Theory, pages 367-380. Academic Press, Toronto, Canada, 1984. (Proc. Waterloo Silver Jubilee Conf. 1982). 
[LV99] M. Las Vergnas. The Tutte polynomial of a morphism of matroids I. Set-pointed matroids and matroid perspectives. Ann. Inst. Fourier, Grenoble, 49(3):973-1015, 1999.

[LV01] M. Las Vergnas. Active orders for matroid bases. Europ. J. Comb., 22:709-721, 2001.

[LV12] M. Las Vergnas. The Tutte polynomial of a morphism of matroids 6. A multi-faceted counting formula for hyperplane regions and acyclic orientations. 2012. Unpublished, preliminary preprint available at arXiv 1205.5424.

[LV13] M. Las Vergnas. The Tutte polynomial of a morphism of matroids 5. Derivatives as generating functions of Tutte activities. Europ. J. Comb., 34:1390-1405, 2013.

[Oxl11] J.G. Oxley. Matroid Theory. Oxford Graduate Texts in Mathematics. Oxford University Press, 2011. Second edition (first edition in 1992).

[Tut54] W.T. Tutte. A contribution to the theory of chromatic polynomials. Canadian J. Math., 6:80-91, 1954. 